\title{
OLIMPÍADA BRASILEIRA DE INFORMÁTICA NO MUNICÍPIO DE VIDEIRA/SC: FERRAMENTA WEB PARA AVALIAR O PERFIL DOS COMPETIDORES
}

http://dx.doi.org/10.5902/2318133835949

\author{
Leila Lisiane Rossi ${ }^{1}$ \\ Luiz Gustavo Moro Senko² \\ Hygor Albert Marques ${ }^{3}$ \\ Maria José de Castro Bonfim ${ }^{4}$
}

\begin{abstract}
Resumo
Neste texto se apresenta um trabalho que teve como objetivo a criação de uma ferramenta que permitisse realizar consultas analíticas online - olaps - sobre os dados dos competidores das escolas de Videira/SC que participaram da Olimpíada Brasileira de Informática - OBI - 2017. A olimpíada de 2017 foi realizada por meio de um projeto de extensão do Instituto Federal Catarinense, campus Videira, com vistas à incentivar as escolas da cidade a participarem dessas provas, que são realizadas seguindo o calendário definido pela Universidade de Campinas, instituição organizadora da OBI. A participação do IFC envolveu a divulgação, aplicação de provas, conferência e o lançamento da pontuação obtida para cada um dos alunos.

Palavras-chave: OBI, olap, perfil.
\end{abstract}

\section{BRAZILIAN OLYMPIAD OF INFORMATIC IN VIDEIRA/SC CITY: WEB TOOL TO MONITOR AND AVALIATE COMPETITORS PROFILE}

\begin{abstract}
This project is aim to create a web tool that allows analytic query online - olaps - about data from schools students in Videira city that participated of the Brazilian Olympiad of Informatic - OBI 2017. The OBI was developed from an extension project of Federal Institute of Santa Catarina - campus Videira, and the main objective was encourage schools of Videira city to participate of these tests that follow the calendar of Campinas University, OBI organizing Institution. IFC's participation is since the dissemination, application, conference and the release of the score obtained for each of competitors.

Keywords: OBI; olap; profile.
\end{abstract}

\footnotetext{
${ }^{1}$ Instituto Federal Catarinense - campus de Videira, Brasil. E-mail: leila.rossi@ifc.edu.br.

2 Instituto Federal Catarinense - campus de Brusque, Brasil. E-mail: luiz.senko@ifc.edu.br.

3 Instituto Federal Catarinense - campus de Videira, Brasil. E-mail: srjota123@outlook.com.

${ }^{4}$ Instituto Federal Catarinense - campus de Videira, Brasil. E-mail: maria.bonfim@ifc.edu.br.

\begin{tabular}{l|lll|l} 
Regae: Rev. Gest. Aval. Educ. & Santa Maria & v. 8 & n. 17 & Pub. contínua 2019
\end{tabular}
} p. 1-8 


\section{Introdução}

participação dos alunos em atividades extracurriculares é importante para a
sua formação. Por meio de projetos abertos à comunidade proporciona-se o
acesso a atividades diferenciadas das que ocorrem no dia a dia em sala de aula, permitindo ao aluno complementar o seu conhecimento em algumas áreas, bem como adquirir novas experiências. E conhecer o perfil dos alunos das mais variadas modalidades de ensino contribui para a escolha de políticas educacionais a serem adotadas.

A OBI na cidade de Videira/SC foi realizada por meio de um projeto de extensão, do qual participaram três escolas e alunos do Instituto Federal Catarinense, campus de Videira, no ano de 2017. Em 2018 cinco escolas participaram e mais alguns alunos do próprio IFC/Videira. Os dados desse ano não estão completos porque a olimpíada ainda está em andamento. $O$ objetivo do presente projeto foi a criação de uma ferramenta web que permitisse identificar o perfil dos competidores da Olimpíada Brasileira de Informática - OBI 2017 (Unicamp, 2018). Esse perfil foi identificado visualizando os dados de forma multidimensional por consultas olap geradas.

\section{Modelo Estrela}

Para a criação da ferramenta web foi realizada a coleta dos dados dos competidores da OBI 2017 a partir do site da Unicamp, organizadora da olimpíada. Na sequência foi efetuado o cadastro dos dados em um banco de dados relacional software livre (Postgresql, 2018). Considerando que o projeto está em andamento, no momento está sendo complementado o modelo multidimensional estrela, figura 1 , o qual permitirá a realização de consultas analíticas online (Pentaho, 2018), para identificar o perfil dos competidores da OBI 2017.

A tabela Fato recebe as chaves das demais tabelas, além de um campo conhecido como Medida, cujo objetivo é realizar operações estatísticas como uma soma ou uma média. A tabela Dimensão, por sua vez, possui informações extraídas do banco de dados relacional e importantes para a realização posterior da olap. A definição das tabelas Dimensão são realizadas na fase de projeto do Data Webhouse, considerando o que deseja-se obter de consulta. A tabela Fato contém as chaves das tabelas Dimensão Pontuação, Sexo, Escola, Fase e Modalidade. Vale destacar que as tabelas Dimensão foram criadas com base nos dados disponíveis e de domínio público sem o acréscimo de informações. 
Figura 1 -

OBI 2017.

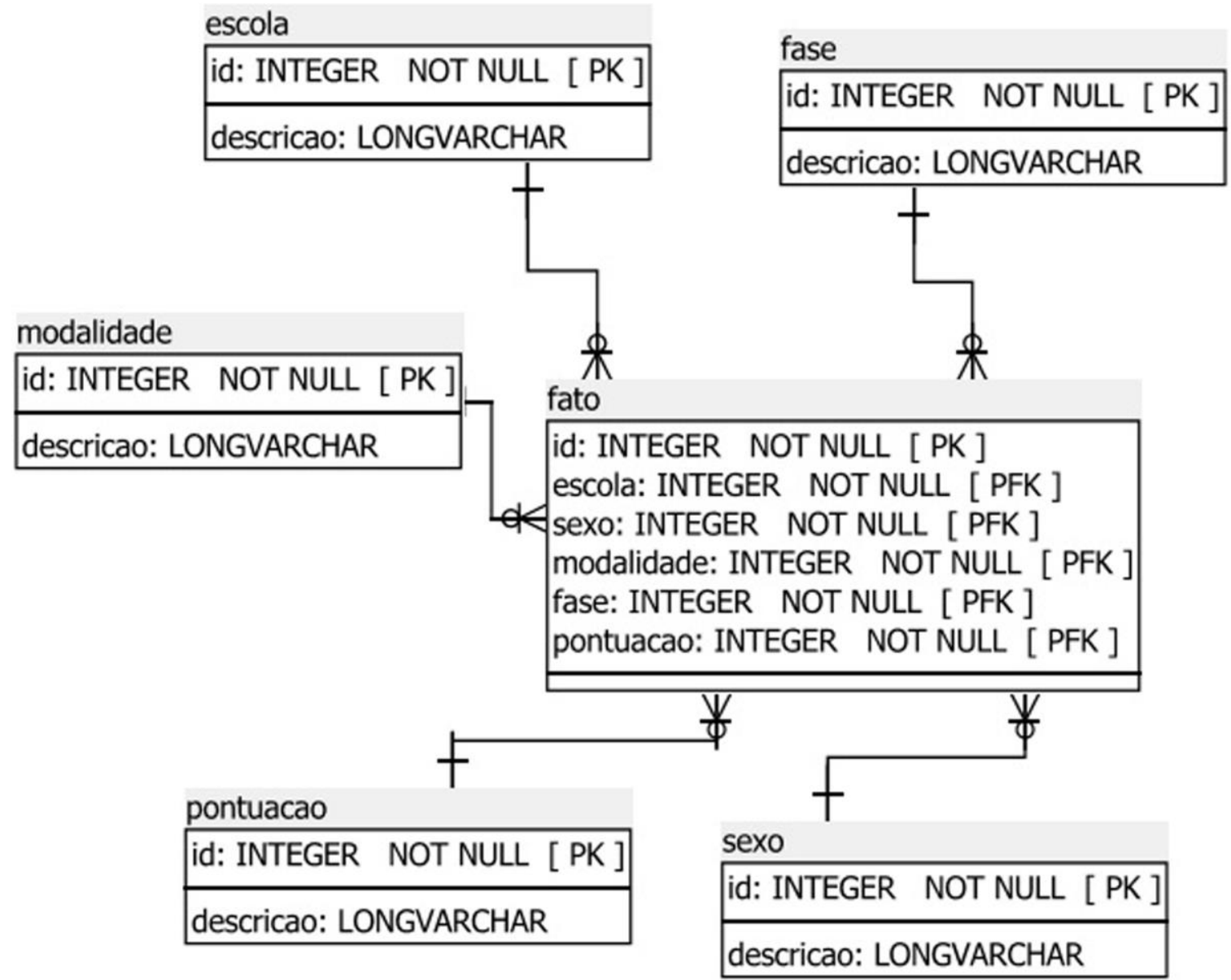

\section{Resultados obtidos}

Os principais resultados obtidos até o momento, considerando que o mesmo se encontra em andamento, são descritos a seguir: após a coleta dos dados do site da Olimpíada Brasileira de Informática (Unicamp, 2018), os mesmos foram carregados no banco de dados relacional e, posteriormente, foi criado o modelo multidimensional Estrela, conforme a figura 1. A partir do modelo multidimensional estrela, foram criadas as primeiras consultas olaps para testar a ferramenta. A figura 3 apresenta um exemplo de consulta olap na qual é possível identificar o número de competidores que participaram da Olimpíada por sexo e por escola. Na ferramenta a consulta pode ser visualizada em mais níveis de detalhamento. No presente trabalho as mesmas foram simplificadas considerando o espaço disponível bem como a qualidade da imagem. 
Figura 2 -

Logo OBI 2017.

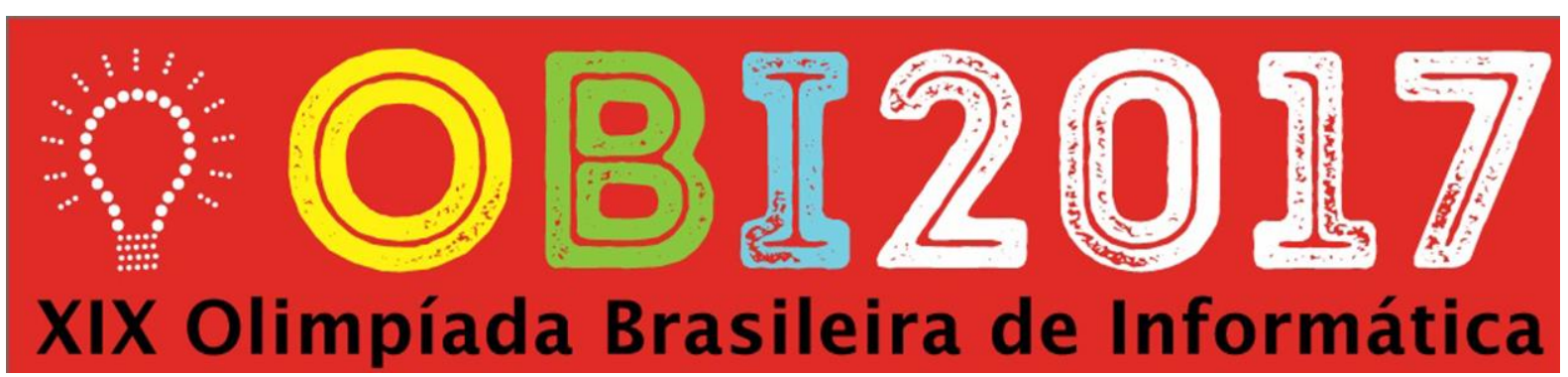

Figura 3 -

Consulta olap Escolas.

\begin{tabular}{|c|c|c|c|c|c|}
\hline escola & fase & modalidade & pontuacao & sexo & $\begin{array}{l}\text { Measures } \\
\text { - total }\end{array}$ \\
\hline$\because$ All escolas & $\oplus_{\text {All fases }}$ & $\oplus_{\text {All modalidades }}$ & $\oplus_{\text {All pontuacaos }}$ & $\oplus_{\text {All sexos }}$ & 404 \\
\hline \multirow[t]{3}{*}{ Joaquim_Amarante } & \multirow[t]{3}{*}{$\oplus_{\text {All fases }}$} & \multirow[t]{3}{*}{$\oplus_{\text {All modalidades }}$} & \multirow[t]{3}{*}{$\oplus_{\text {All pontuacaos }}$} & $\square_{\text {All sexos }}$ & 256 \\
\hline & & & & Feminino & 122 \\
\hline & & & & Masculino & 134 \\
\hline \multirow[t]{3}{*}{ Vilson_Kleinubing } & \multirow[t]{3}{*}{$\oplus_{\text {All fases }}$} & \multirow[t]{3}{*}{$\oplus_{\text {All modalidades }}$} & \multirow[t]{3}{*}{$\oplus_{\text {All pontuacaos }}$} & $\nabla_{\text {All sexos }}$ & 78 \\
\hline & & & & Feminino & 28 \\
\hline & & & & Masculino & 50 \\
\hline \multirow[t]{3}{*}{ Waldemar_Kleinubing } & \multirow[t]{3}{*}{$\oplus_{\text {All fases }}$} & \multirow[t]{3}{*}{${ }^{\dagger}$ All modalidades } & \multirow[t]{3}{*}{$\oplus_{\text {All pontuacaos }}$} & $\nabla_{\text {All sexos }}$ & 70 \\
\hline & & & & Feminino & 28 \\
\hline & & & & Masculino & 42 \\
\hline
\end{tabular}

Percebe-se que o número de participantes de ambos os sexos são muito próximos, sendo que o masculino é ligeiramente maior. Esse resultado é um indicativo de que a divulgação do projeto é uma fase muito importante e que deve ser feita com planejamento e organização facilitando assim o alcance dos objetivos finais. 
Figura 4 -

Consulta olap Fases.

\begin{tabular}{|c|c|c|c|c|c|}
\hline & & & & & Measures \\
\hline escola & fase & modalidade & pontuacao & sexo & - total \\
\hline$\oplus_{\text {All escolas }}$ & $\nabla_{\text {All fases }}$ & All modalidades & $\oplus_{\text {All pontuacaos }}$ & $\oplus_{\text {All sexos }}$ & 404 \\
\hline & & Iniciacao_1 & $\oplus_{\text {All pontuacaos }}$ & $\oplus_{\text {All sexos }}$ & 240 \\
\hline & & Iniciacao_2 & $\oplus_{\text {All pontuacaos }}$ & $\oplus_{\text {All sexos }}$ & 164 \\
\hline & Estadual & All modalidades & $\oplus_{\text {All pontuacaos }}$ & $\oplus_{\text {All sexos }}$ & 84 \\
\hline & & Iniciacao_1 & $+_{\text {All pontuacaos }}$ & $\oplus_{\text {All sexos }}$ & 55 \\
\hline & & Iniciacao_2 & $\oplus_{\text {All pontuacaos }}$ & $\pm_{\text {All sexos }}$ & 29 \\
\hline & Local & All modalidades & $\oplus_{\text {All pontuacaos }}$ & $\oplus_{\text {All sexos }}$ & 298 \\
\hline & & Iniciacao_1 & $\oplus_{\text {All pontuacaos }}$ & $\oplus_{\text {All sexos }}$ & 169 \\
\hline & & Iniciacao_2 & $\oplus_{\text {All pontuacaos }}$ & $\oplus_{\text {All sexos }}$ & 129 \\
\hline & Nacional & All modalidades & $\oplus_{\text {All pontuacaos }}$ & $\oplus_{\text {All sexos }}$ & 22 \\
\hline & & Iniciacao_1 & $\oplus_{\text {All pontuacaos }}$ & + All sexos & 16 \\
\hline & & Iniciacao_2 & $\oplus_{\text {All pontuacaos }}$ & $\oplus_{\text {All sexos }}$ & 6 \\
\hline
\end{tabular}

Figura 5 -

Tela Ferramenta Mineração de Dados Weka.

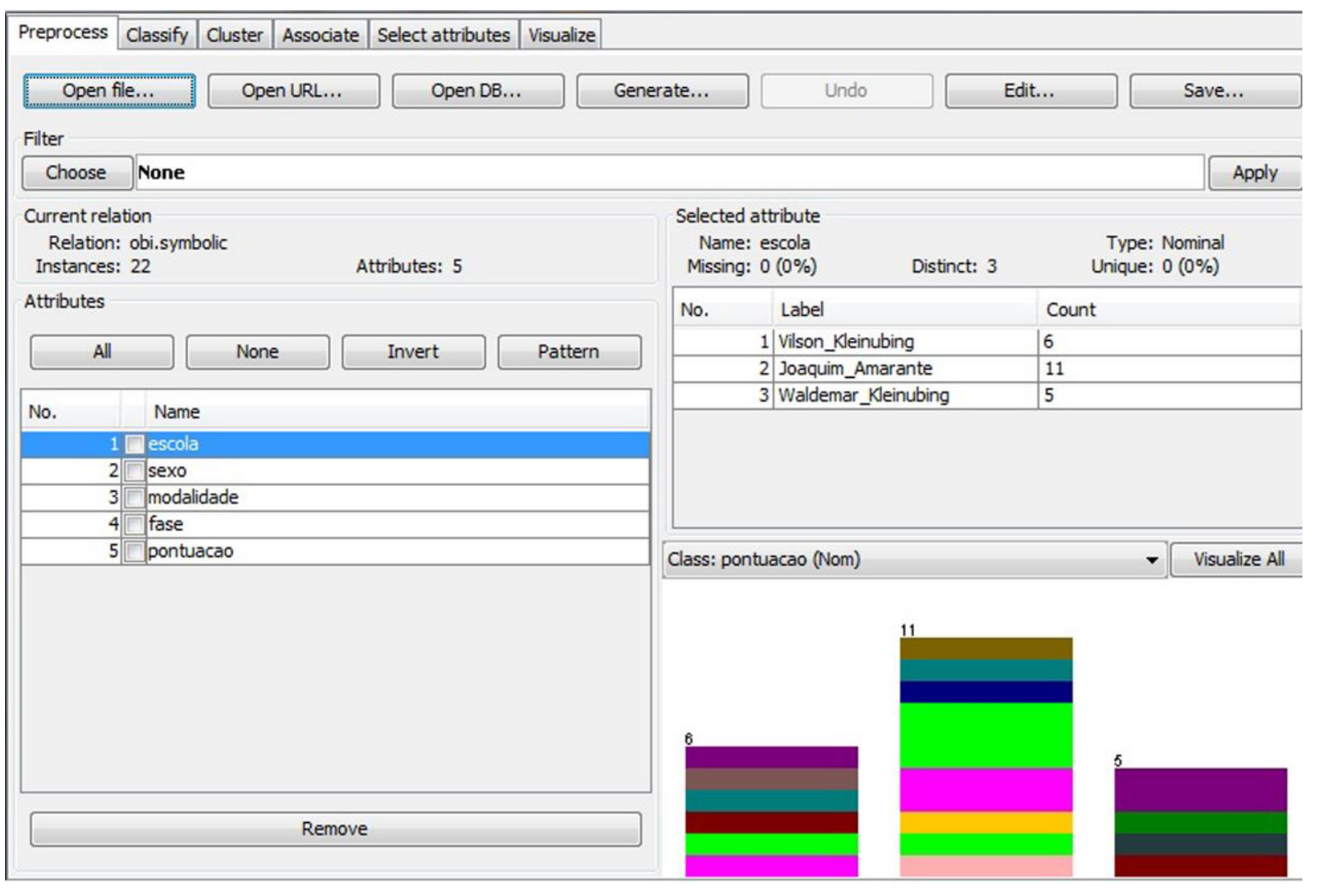


$\mathrm{Na}$ figura 4 é apresentado um outro de exemplo de consulta olap na qual é possível identificar o número de competidores por fase e por modalidade. Por exemplo, para a fase nacional, na modalidade Iniciação 2 , foram classificados seis competidores. $\mathrm{Na}$ ferramenta é possível detalhar melhor permitindo a visualização dos dados por escola, sexo, fase, modalidade e pontuação obtida. A figura 5 apresenta uma tela da ferramenta de mineração de dados Weka (Pentaho, 2018), a partir da qual é possível selecionar os dados como a escola, a fase, entre outros, gerando vários gráficos como resultado. Os principais algoritmos de mineração de dados que foram executados pela ferramenta são os de associação e agrupamento ou cluster, conforme a figura 6 , a qual apresenta dois clusters gerados pelo algoritmo SimpleKMeans. Um deles mostra os dados agrupados da Escola Joaquim Amarante e outro da Escola Vilson Pedro Kleinubing.

Figura 6 -

Tela cluster gerado pela Weka.

Cluster centroids:

$\begin{array}{lrrr}\text { Attribute } & \text { Full Data } & \text { Cluster\# } & 1 \\ & (22) & 0 & (10) \\ \text { e============================================================ } \\ \text { escola } & \text { Joaquim_Amarante Vilson_Kleinubing } & \text { Joaquim_Amarante } \\ \text { sexo } & \text { feminino } & \text { masculino } & \text { feminino } \\ \text { modalidade } & \text { Iniciacao_1 } & \text { Iniciacao_1 } & \text { Iniciacao_1 } \\ \text { fase } & \text { Nacional } & \text { Nacional } & \text { Nacional } \\ \text { pontuacao } & 9 & 17 & 9\end{array}$

\section{Conclusão e trabalhos futuros}

O perfil dos alunos das mais variadas modalidades de ensino são importantes na tomada de decisão em uma instituição de ensino. No caso específico do presente projeto espera-se obter uma ferramenta web com a interface amigável e que permita a realização de consultas OLAPs sobre os dados da OBI 2017 identificando, por exemplo, a pontuação obtida em cada fase, por escola, sexo, modalidade e nível. Com base nos resultados e conforme disponibilidade do instituto e interesse das escolas, poderão ser propostos cursos preparatórios de lógica e programação aos alunos interessados ou para os mais bem colocados como forma de premiação e incentivo.

Vale destacar que o trabalho ficou classificado em segundo lugar como projeto de pesquisa do ensino médio em andamento na VII Feira de Iniciação Científica e Extensão. Como forma de incentivo e considerando que a o resultado da etapa nacional 2018 ainda não havia sido divulgado, os alunos com melhor pontuação na fase estadual das escolas e do IFC/Videira, que realizaram a prova nacional, receberam uma medalha do próprio Instituto Federal Catarinense durante a VII Fice realizada no campus Videira. 


\section{Referências}

KIMBALL, Ralph. Data warehouse toolkit: o guia completo para modelagem multidimensional. Rio de Janeiro: Campus, 2002.

ELMASRI, Ramez; NAVATHE, Shamkant B. Sistemas de banco de dados. São Paulo: Pearson/Addison Wesley, 2011.

MILANI, André. PostgreSQL: guia do programador. São Paulo: Novatec, 2008.

PENTAHO. Pentaho open source business intelligence. Disponível em http://www.pentaho.com. Acesso em 18 nov. 2018.

POSTGRESQL. PostgreSQL. Disponível em http://www.postgresql.org. Acesso em 18 nov. 2018.

PRENSKY, Marc. Digital natives, digital immigrants. On the Horizon, NCB University Press, $\quad$ v. $9, \quad$ n. $5, \quad 2001.20$ Disponível http://webcache.googleusercontent.com/search?q=cache:uZh3Ue46ycAJ:www.marvidar.c om/wp-content/uploads/Digital_Natives_by_Marc_Prensky1.doc+\&cd=3\&hl=pt-

PT\&ct=clnk\&gl=br. Acesso em 18 nov. 2018

SILBERSCHATZ, Abraham; KORTH, Henry F; SUDARCHAN, S. Sistemas de banco de dados. São Paulo: Makron Books, 2006.

UNICAMP. Olimpíada Brasileira de Informática - OBI. Disponível em http://olimpiada.ic.unicamp.br. Acesso em 18 nov. 2018

WEKA, Pentaho. Open source business intelligence - data mining. Disponível em http://community.pentaho.com/projects/data-mining. Acesso em 18 nov. 2018.

WITTEN, Ian H; FRANK, Eibe. Data mining: practical machine learning tools and techniques. San Francisco: Elsevier, 2005.

Leila Lisiane Rossi é mestre em Ciência da Computação e professora de Informática no Instituto Federal Catarinense, campus de Videira.

Orcid: https://orcid.org/0000-0002-4668-2735.

Endereço: Instituto Federal Catarinense - campus de Videira - rodovia SC 135, Km 125 - 89560-000 - Videira - SC - Brasil.

E-mail: leila.rossi@ifc.edu.br.

Luiz Gustavo Moro Senko é mestre em Ciência da Computação e professor de Informática no Instituto Federal Catarinense, campus de Brusque.

Orcid: https://orcid.org/0000-0001-8308-3056.

Endereço: Instituto Federal Catarinense - campus de Brusque - Rua Hercílio Luz, 373 - 88350-301 - Brusque - SC - Brasil.

E-mail: luiz.senko@ifc.edu.br. 
Hygor Albert Marques é estudante no curso Técnico Integrado em Informática e bolsista do projeto de pesquisa no Instituto Federal Catarinense, campus de Videira.

Endereço: Instituto Federal Catarinense - campus de Videira - rodovia SC 135, Km 125 - 89560-000 - Videira - SC - Brasil.

Orcid: https://orcid.org/0000-0002-1171-7555.

E-mail: srjota123@outlook.com.

Maria José de Castro Bonfim é especialista em Marketing, bacharel em Design Gráfico e programadora visual no Instituto Federal Catarinense, campus de Videira.

Orcid: https://orcid.org/0000-0003-0115-1452.

Endereço: Instituto Federal Catarinense - rodovia SC 135, Km 125 - 89560-000 Videira - SC - Brasil.

E-mail: maria.bonfim@ifc.edu.br.

Recebido em 6 de dezembro de 2018.

Aprovado em 4 de janeiro de 2019.

(c) (i) 